\title{
Modeling the observed proton aurora and ionospheric convection responses to changes in the IMF clock angle: 1. Persistence of cusp proton aurora
}

Article

Published Version

Throp, K., Lockwood, M., Lanchester, B. S., Morley, S. K. and Frey, H. U. (2005) Modeling the observed proton aurora and ionospheric convection responses to changes in the IMF clock angle: 1. Persistence of cusp proton aurora. Journal of Geophysical Research, 110 (A12). A12311. ISSN 0148-0227 doi: https://doi.org/10.1029/2003JA010306 Available at https://centaur.reading.ac.uk/38591/

It is advisable to refer to the publisher's version if you intend to cite from the work. See Guidance on citing.

Published version at: http://dx.doi.org/10.1029/2003JA010306

To link to this article DOI: http://dx.doi.org/10.1029/2003JA010306

Publisher: American Geophysical Union

All outputs in CentAUR are protected by Intellectual Property Rights law, including copyright law. Copyright and IPR is retained by the creators or other copyright holders. Terms and conditions for use of this material are defined in the End User Agreement. 


\section{www.reading.ac.uk/centaur}

\section{CentAUR}

Central Archive at the University of Reading

Reading's research outputs online 


\title{
Modeling the observed proton aurora and ionospheric convection responses to changes in the IMF clock angle: 1. Persistence of cusp proton aurora
}

\author{
K. Throp, M. Lockwood, ${ }^{1}$ B. S. Lanchester, and S. K. Morley ${ }^{2}$ \\ Department of Physics and Astronomy, Southampton University, Southampton, Hampshire, UK
}

H. U. Frey

Space Sciences Laboratory, University of California, Berkeley, Berkeley, California, USA

Received 27 October 2003; revised 27 August 2005; accepted 5 October 2005; published 21 December 2005.

[1] We employ a numerical model of cusp ion precipitation and proton aurora emission to fit variations of the peak Doppler-shifted Lyman- $\alpha$ intensity observed on 26 November 2000 by the SI-12 channel of the FUV instrument on the IMAGE satellite. The major features of this event appeared in response to two brief swings of the interplanetary magnetic field (IMF) toward a southward orientation. We reproduce the observed spatial distributions of this emission on newly opened field lines by combining the proton emission model with a model of the response of ionospheric convection. The simulations are based on the observed variations of the solar wind proton temperature and concentration and the interplanetary magnetic field clock angle. They also allow for the efficiency, sampling rate, integration time and spatial resolution of the FUV instrument. The good match (correlation coefficient 0.91, significant at the $98 \%$ level) between observed and modeled variations confirms the time constant ( $\sim 4 \mathrm{~min}$ ) for the rise and decay of the proton emissions predicted by the model for southward IMF conditions. The implications for the detection of pulsed magnetopause reconnection using proton aurora are discussed for a range of interplanetary conditions.

Citation: Throp, K., M. Lockwood, B. S. Lanchester, S. K. Morley, and H. U. Frey (2005), Modeling the observed proton aurora and ionospheric convection responses to changes in the IMF clock angle: 1. Persistence of cusp proton aurora, J. Geophys. Res., 110, A12311, doi:10.1029/2003JA010306.

\section{Introduction}

[2] The advent of space-based imagers observing the Earth in Doppler-shifted Lyman- $\alpha$ emissions has drawn attention to the use of the global distribution of proton aurora to identify newly opened field lines generated by magnetopause reconnection. Precipitations of solar wind electrons and ions, guided along newly reconnected field lines across the magnetopause and into the cusp region, cause the excitation of auroral emissions. While not the main source of particle energy flux associated with these emissions, protons have relatively long flight times and, as a result, they carry information about the recent history of the reconnection process [Lockwood and Smith, 1992]. In addition, the precipitation of electrons is greatly influenced by the proton flux as quasi-neutrality is maintained along the field lines [Burch, 1985] and significant contributions to

\footnotetext{
${ }^{1}$ Also at Rutherford Appleton Laboratory, Chilton, Oxfordshire, UK. ${ }^{2}$ Now at CRC for Satellite Systems, University of Newcastle, New South Wales, Australia.

Copyright 2005 by the American Geophysical Union. 0148-0227/05/2003JA010306\$09.00
}

the emission of "electron" aurora are made by secondary electrons generated by precipitating protons.

[3] Lockwood et al. [2003] (hereafter referred to as LEA03) reported an event on the 26 November 2000 in which a double brightening of Lyman- $\alpha$ emitted from the dayside cusp was observed by the SI-12 channel of the FUV instrument on the IMAGE satellite [Mende et al., 2000a, 2000b; Frey et al., 2001a, 2001b]. This double brightening occurred in response to two short-lived southward rotations of the Interplanetary Magnetic Field (IMF), during a period of strongly enhanced solar wind plasma concentration. Application of derived propagation lags revealed that the peak emission intensity varied systematically with the upstream IMF clock angle. This association is demonstrated by comparison of Figures 1a and 1c, adapted from LEA03, which summarizes the conditions prevailing during this event. The agreement of the IMF clock angle with the peak observed proton aurora intensity was found to be considerably stronger if the latter was normalized to allow for the variation in the solar wind concentration, shown in Figure $1 b$.

[4] The changes in the spatial distribution of proton aurora during the event, as reported by LEA03, are shown in Figure 2, along with the pattern of ionospheric convec- 


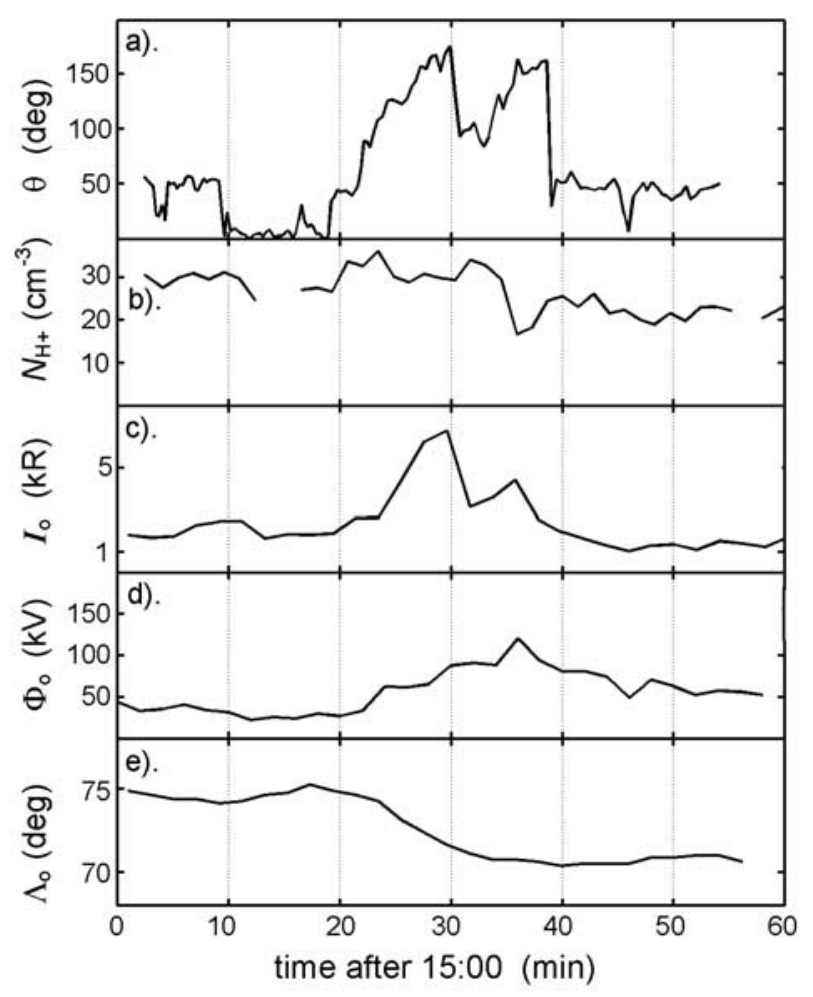

Figure 1. Variations of parameters observed during the brief double intensification of the cusp proton aurora on 26 November 2000 reported by LEA03: (a) the lagged IMF clock angle $\theta$, and (b) the lagged proton concentration in the solar wind $\mathrm{N}_{\mathrm{H}+}$, as observed by ACE; (c) the peak Dopplershifted Lyman- $\alpha$ emission intensity seen by FUV/SI-12, $I_{\mathrm{o}}$; (d) the transpolar voltage derived from the SuperDARN radar data $\Phi_{\mathrm{o}}$; and (e) the observed latitude of the equatorward edge of the electron aurora seen in OI emissions by the SI-13/FUV instrument on IMAGE, $\Lambda_{\mathrm{o}}$. The solar wind and IMF data have been lagged by the ACEto-ionosphere propagation delay variation $\delta t_{\mathrm{E}}\left(\mathbf{t}_{\mathrm{s}}\right)$ for this interval, as obtained by LEA03 from comparisons of WIND and ACE data.

tion, derived from SuperDARN HF radar observations, using the method of Ruohoniemi and Baker [1998]. By analyzing particle data from passes of DMSP (Defense Meterological Satellite Program) spacecraft, LEA03 confirmed that the variable bright spot in the proton aurora around noon was the cusp region. In this paper, we present model simulations of the proton aurora data shown in Figure 2. In a companion paper [Lockwood et al., 2005, hereafter referred to as Paper II] we present simulations of the convection data shown in Figure 2 and Figure 1d.

[5] Ions injected into the magnetosphere are accelerated as they cross the current layer of the dayside magnetopause [Cowley, 1982] and the effect of this acceleration is apparent in both observations and numerical simulations of the dispersed precipitation of ions into the cusp ionosphere [Hill and Reiff, 1977; Lockwood and Smith, 1992; Onsager et al., 1993; Lockwood, 1997; Lockwood and Hapgood, 1998]. In LEA03, the numerical cusp model developed by
Lockwood and Davis [1996] and Lockwood [1997] was generalized to replicate the effect on the acceleration of variations in the sheath field clock angle. The original model of Lockwood and Davis allowed only for the special case where the sheath-field and interior magnetospheric fields were coplanar (i.e., the sheath field clock angle, $\theta_{\mathrm{sh}}=180^{\circ}$ ), known as "antiparallel reconnection" [Crooker, 1979; Chisham et al., 2002; Coleman et al., 2000, 2001]. The revised model used by LEA03 allows for a range of clock angles, $\theta_{\mathrm{sh}}$, and so can also be used to also model the effects of "component reconnection" (for which $\theta_{\mathrm{sh}}$ can be lower than $180^{\circ}$ ) [Sonnerup, 1974; Gonzalez and Mozer, 1974].

[6] In an attempt to distinguish between antiparallel and component reconnection, LEA03 analyzed the variation of the peak observed intensity, $I_{\mathrm{o}}$, with the interplanetary clock angle $\theta$. The problem with this analysis is that the time-offlight of the ions from the magnetopause to the ionosphere means that the intensity does not respond instantaneously to clock angle changes or to changes in the solar wind concentration $N_{\mathrm{SW}}$. Further complications arise from the dispersed effect of such changes on the precipitating ion spectrum arriving in the cusp ionosphere and the fact that the efficiency of proton aurora excitation is a function of ion energy [Gérard et al., 2001]. In addition, the IMAGE FUV instrument resolves proton aurora from geocoronal emissions by accepting only Doppler-shifted Lyman- $\alpha$, making the instrument response also highly dependent on ion energy. One result is that the delay of peak emission after reconnection is a function of clock angle $\theta$ (see LEA03, Figure 15) and thus, for example, during a period of low $N_{\mathrm{SW}}$ and reduced $\theta$, the peak intensity observed $I_{\mathrm{o}}$ may be higher than expected, resulting from field lines opened at an earlier reconnection time when $N_{\mathrm{Sw}}$ and/or $\theta$ were larger. In the present paper, we repeat the analysis of LEA03 allowing for these effects. This is achieved by predicting the variation of intensity on the newly opened field lines, allowing for the observed variations in $N_{\mathrm{SW}}$ and $\theta$ and convolving the results with the FUV/SI-12 instrument response and sampling. Thus the simulation exactly parallels the production of the measured intensity data and the peak simulated intensity, $I_{\mathrm{m}}$, can be compared with the peak intensity $I_{\mathrm{o}}$ measured by the FUV/SI-12 instrument.

[7] A parallel study presented in Paper II uses the numerical model of Lockwood and Morley [2004] (hereafter LM04), to predict the evolution of ionospheric convection flows in response to the observed IMF changes during this event. This is done using a novel application of the LM04 model in which the time-dependent magnetopause reconnection rate, an input to the model, is specified by the observed upstream IMF conditions. In Paper II, the predicted variations in the latitude of the open-closed field-line boundary, $\Lambda_{\mathrm{OCB}}$, and of the transpolar voltage, $\Phi_{\mathrm{PC}}$, are compared to the observations (by the IMAGE satellite and the SuperDARN radar network, respectively, and shown in Figures 1e and 1d). We here use the convection simulations given in Paper II to predict the spatial distribution of proton aurora emission.

[8] Recently, Frey et al. [2003] have presented observations by FUV/SI-12 showing persistent and quasi-steady proton aurora during prolonged $(\sim 10$ hour $)$ periods of northward IMF. If the proton aurora was an immediate 



Figure 2. Global images of the Doppler-shifted Lyman- $\alpha$ emission seen by the SI-12 channel of the FUV instrument on the IMAGE spacecraft, with superposed convection potential contours derived from observations by the SuperDARN HF coherent radar network. The images are 5-s integrations, taken once every $122 \mathrm{~s}$. The intensity scale is the same in each frame (absolute intensities are given by the scale in Figure 8). The convection patterns are produced by the mapped-potential technique in which a model is fitted to all line-of-sight velocity observations, the model used being determined by the IMF orientation seen by ACE for best estimates of the ACE-ionosphere lag, $\delta t_{\mathrm{E}}$. For each image, the closest 90 -s radar scan has been employed. Streamlines are $6 \mathrm{kV}$ apart. Above each frame is given the time of the FUV image, the start time of the radar scan, and (in parentheses) the lag $\delta t_{\mathrm{E}}$ employed. The vector in the top right of each frame is the lagged IMF in the $\left[B_{Z}\right]_{\mathrm{GSM}}$ (up the page) $-\left[B_{\mathrm{Y}}\right]_{\mathrm{GSM}}$ (to the right) frame, the circle corresponding to a magnitude of $15 \mathrm{nT}$. 
response to the reconnection, these observations would eliminate the possibility of intervals when the reconnection rate fell to zero and so would show that the reconnection was continuous in nature. However, because of the rise and fall times of the proton aurora response to reconnection, the observations only place limits on any periods of zero reconnection rate. Nevertheless, these are tighter constraints than for the more long-lived emissions caused by cusp electron precipitation. Cusp electrons are usually undispersed and persist down each newly opened field line for an extended period following reconnection, as the field line evolves from the reconnection site into the tail lobe. In particular, the much-studied $630 \mathrm{~nm}$ oxygen emission is thermally excited by enhanced ionospheric temperatures caused by cusp precipitation and has a long ( $\sim 2 \mathrm{~min})$ deexcitation time. These factors result in this "red-line" emission persisting for of order $10 \mathrm{~min}$ around the footprint of each newly opened field line and the effects of a pulse reconnection will be smoothed with such a time constant [Lockwood and Davis, 1995; Davis and Lockwood, 1996]. The proton emission seen by FUV/SI-12, on the other hand, requires the more energetic of the dispersed cusp ions which persist for shorter intervals on each newly opened field line, and thus periods of zero reconnection [Lockwood et al., 1998] should be easier to detect. In the present paper, we compute and verify the time constants for the growth and decay of proton emission intensity, as seen by FUV/SI-12, and investigate the implications for observing pulsed reconnection effects during intervals of southward IMF.

\section{Modeling the Temporal and Spatial Variations of the Proton Aurora}

[9] Figure 3 summarizes the operation of the cusp ion precipitation model used in this paper. Because it employs output from the convection model, labels for operations, inputs and outputs follow on from those for the convection model described by the corresponding flowchart in Figure 1 of Paper II. The model follows the evolution of the both the ionospheric footpoint, F, and of the magnetopause threading point, M, of one newly opened field line and hence studies the evolution of the precipitating proton spectrum arriving at $\mathrm{F}$ as a function of time elapsed since reconnection. It requires a number of inputs. I.5 is the observed upstream solar wind density, temperature, and velocity. I.7 is a pair of empirical models. The best fit of the axisymmetric magnetopause shape used by Spreiter et al. [1966] is fitted to the Sibeck et al. [1991] magnetopause model for the prevailing solar wind conditions, so defining the relationship between the three components of the geocentric position vector of the boundary location, $\mathbf{R}_{\mathrm{M}}$. (Note that this shape is used as it allows us to employ the gas-dynamic sheath simulations by Sprieter et al.). The Tsyganenko [1987] T87 model is used to give the magnetospheric field, with its magnetopause arranged to be everywhere just outside the model magnetopause used so that from every magnetopause location $\mathrm{M}$ a field-aligned distance to the ionosphere, $\mathrm{d}$ can be calculated and hence the time of flight $\delta \mathrm{t}$ of an ion of given energy is known. Each run of the model is for a single initial MLT on the reconnection $\mathrm{X}$-line, $[\mathrm{MLT}]_{\mathrm{M}}\left(t_{\mathrm{s}}=0\right)$, and so the model is repeatedly rerun for a range of $[\mathrm{MLT}]_{\mathrm{M}}\left(t_{\mathrm{s}}=0\right)$ to cover the full X-line extent.
[10] The longitudinal structure in the magnetosheath means that the concentration and temperatures of the plasma crossing the magnetopause are functions of MLT. In order to reproduce the consequent longitudinal structure in the ionosphere, we need to map this sheath structure down the field lines to the ionosphere. In general, the mapping of the magnetopause $\mathrm{X}$-line into the ionospheric merging gap depends upon the amount of open flux present [Crooker et al., 1991]. We here define the mapping factor by setting an upper limit to the magnetopause reconnection rate $E_{\mathrm{X}}$ of $1 \mathrm{mV} \mathrm{m}^{-1}$ and, because field lines evolve away from the X-line at speeds $V_{\mathrm{F}}$ exceeding $250 \mathrm{~km} \mathrm{~s}^{-1}$, this sets an upper limit to the boundary-normal field of $B_{\mathrm{n}}=E_{\mathrm{X}} / V_{\mathrm{F}} \leq$ $4 \mathrm{nT}$. We obtain the mapping factor by comparing this with the peak electric field in the ionosphere, $E_{\text {no }}$, which is estimated to be $108 \mathrm{mV} \mathrm{m}^{-1}$ in Paper II. (The length $\mathrm{dl}_{\mathrm{x}}$ along the $\mathrm{X}$-line which corresponds to $\mathrm{dl}_{\mathrm{i}}$ along the ionospheric merging gap is given by $\mathrm{E}_{\mathrm{X}} \mathrm{dl}_{\mathrm{X}}=E_{\mathrm{no}} \mathrm{dl}_{\mathrm{i}}$ ). For a magnetopause at a geocentric distance of $12 R_{E}$ and a merging gap at invariant latitude $\Lambda=75^{\circ}$, the resultant mapping factor means that 1 hour of MLT along the ionospheric merging gap maps to approximately 2.5 hours of MLT along the X-line. This way, the initial MLT of the footprint of the reconnection site, $[\mathrm{MLT}]_{\mathrm{F}}\left(t_{\mathrm{s}}=0\right)$, is mapped from $[\mathrm{MLT}]_{\mathrm{M}}\left(t_{\mathrm{S}}=0\right)($ Step $\mathrm{K})$. The initial latitude of the footprint $\Lambda_{\mathrm{F}}\left(t_{\mathrm{s}}=0\right)$ is taken from the convection model predictions (I.4). Subsequent locations of the footprint are known as a function of time $t_{\mathrm{s}}$ because input I.4 gives the ionospheric flow speed and streamline (Step R). The gasdynamic predictions Spreiter et al. [1966] of sheath flow and associated magnetic field draping are used to give the sheath number density, temperature, flow velocity vector and Alfvén velocity vector $\left(\mathrm{N}_{\mathrm{SH}}, \mathrm{T}_{\mathrm{SH}}, \mathbf{V}_{\mathrm{SH}}\right.$ and $\mathbf{V}_{\mathrm{A}}$, respectively) close to each point on the boundary (step L). The velocity of the point $\mathrm{M}$ is the evaluated using the Whalén relation; specifically, the field line moves relative to the sheath flow to make plasma flow into the RD at the local Alfvén speed in the de-Hoffman Teller frame (step M). The theory of Cowley [1982], as implemented by Lockwood [1995b], is then used (step N) to evaluate the field-aligned part of the proton differential energy spectrum, $\mathrm{J}_{\mathrm{EM}}\left(\mathrm{E}, \mathrm{t}_{\mathrm{s}}\right)$, injected at $\mathrm{M}$ where $\mathrm{E}$ is proton energy. (Note that it is assumed here that the sheath proton temperature equals the gas-dynamic plasma temperature $\mathrm{T}_{\mathrm{SH}}$ ). The model calculates the next position of the point $\mathrm{M}$ (step P) and then steps forward in time (step Q). Using Loiuville's theorem, the precipitating differential energy flux at the ionosphere can be calculated, allowing for the time of flight $\delta \mathrm{t}$ of a given energy $\mathrm{J}_{\mathrm{EF}}\left(\mathrm{E}, \mathrm{t}_{\mathrm{s}}\right)=\mathrm{J}_{\mathrm{EM}}\left(\mathrm{E}, \mathrm{t}_{\mathrm{s}}-\delta \mathrm{t}\right.$ ) (Output $\left.\mathrm{O} .4\right)$. The precipitating differential energy flux spectrum is also converted into the intensity of the Doppler-shifted Lyman- $\alpha$ emission, convolved with the IMAGE FUV SI-12 instrument response, using the efficiency spectrum predicted by Gérard et al. [2001], as described by LEA03 (Output O.5).

\section{Peak Intensity of the Proton Aurora}

[11] As described above, the model calculates the energy spectra of the precipitating proton population as a function of time since the field line was reconnected (Output O.4), and from these, makes estimates of the resulting Lyman- $\alpha$ emission intensity, convolved with the IMAGE FUV/SI-12 


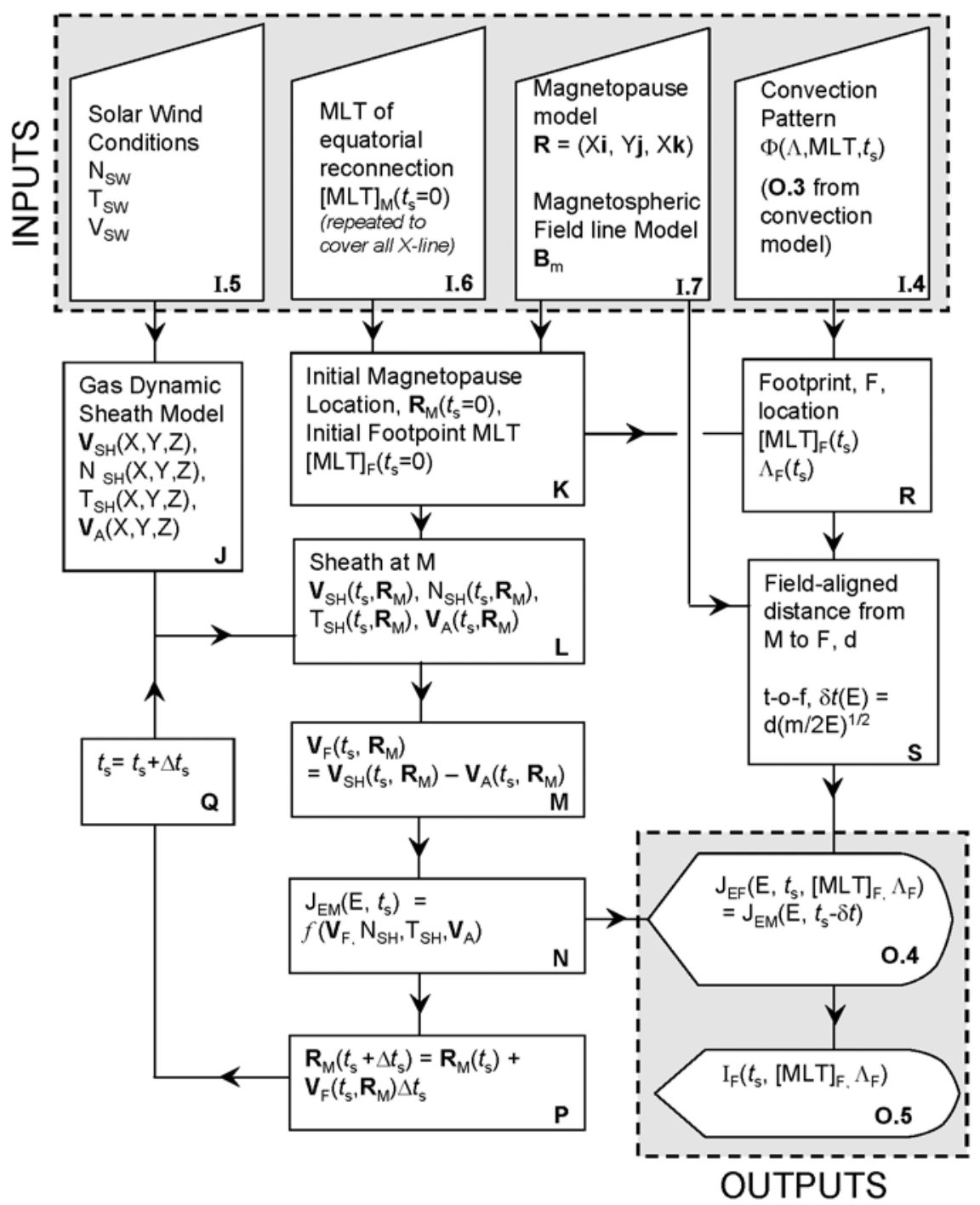

Figure 3. Flowchart of the model of time-dependent cusp auroral proton precipitation, defining the inputs, operations, and outputs, which have been labeled following on from the similar flowchart for the model for the convective flows described in Paper II (see Figure 1 of Paper II). The inputs for the auroral model are the solar wind conditions (input I.5), the location of the reconnection site (I.6), empirical models for the magnetopause location and the internal magnetospheric magnetic field (I.7), and the pattern of ionospheric convection, which is taken from output $\mathrm{O} .3$ of the time-dependent convection model described in Paper II. Gas dynamic modeling of the magnetosheath is used to predict the sheath parameters from the input solar wind observations (operation $\mathrm{J}$ ) and step $\mathrm{K}$ defines the footprint of the reconnection site and the initial position vector, $\mathbf{R}_{\mathrm{M}}\left(\mathrm{t}_{\mathrm{s}}=0\right)$, of the point $\mathrm{M}$ where the field line threads the magnetopause. The location of the footprint of this field line F, as it evolves after reconnection, is evaluated from the model streamlines (input I.4) by the operation R. The field-aligned distance $\mathrm{d}$ between the point $\mathrm{M}$ and the ionosphere is estimated using the magnetospheric magnetic field model in step $\mathrm{S}$, which also calculates the time-of-flight $\delta t$ ions from $\mathrm{M}$ to the ionosphere as a function of their energy, E. Step L draws in the sheath parameters at the point M. Step M calculates the field line velocity vector $\mathbf{V}_{\mathrm{F}}$ in the boundary plane which satisfies the Whalén relation. The differential energy flux spectrum of fieldparallel injected ions at $\mathrm{M}, \mathrm{J}_{\mathrm{EM}}\left(\mathrm{E}, \mathrm{t}_{\mathrm{s}}\right)$ is then computed in step $\mathrm{N}$ using the theory of Cowley [1982] and Lockwood [1995b]. The position vector of the point $\mathrm{M}$ is then calculated from $\mathbf{V}_{\mathrm{F}}$ for the subsequent simulation time (step P), and simulation time advanced (step Q) so the procedure can be repeated. After step $N$ the differential energy flux spectrum of ions appearing at $F, J_{E F}\left(E, t_{s}\right)=J_{E M}\left(E, t_{s}-\delta t\right)$ is computed, allowing for the time delay due to the ions' propagation time $\delta$ t between $\mathrm{M}$ and $\mathrm{F}$ (Output O.4). The Doppler-shifted Lyman- $\alpha$ intensity at $F, \mathrm{I}_{\mathrm{F}}$, convolved with the IMAGE FUV/SI-12 instrument response is then computed using the modeling by Gérard et al. [2001] (Output O.5). The model is repeatedly rerun to cover the full MLT extent of the X-line. 

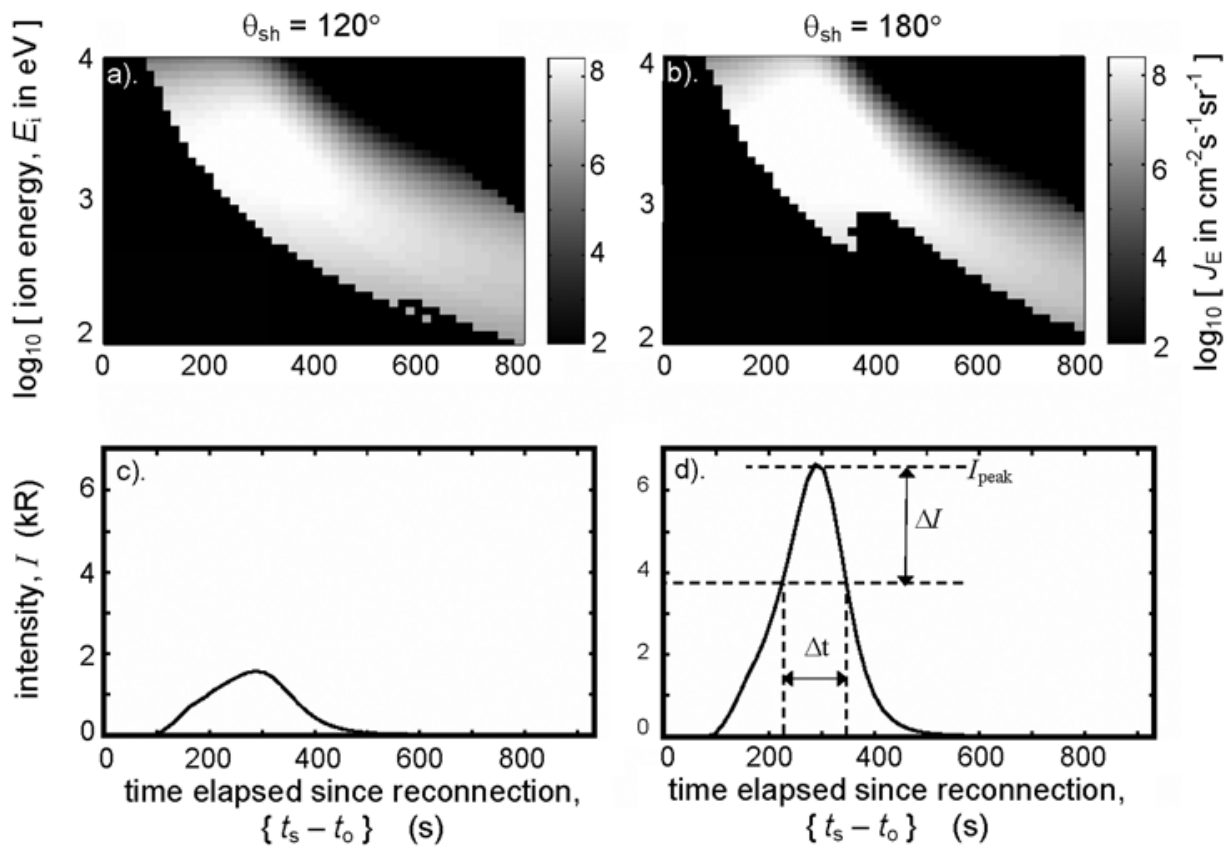

Figure 4. (a) and (b). Modeled variations of cusp ion precipitation down a single newly opened field line reconnected at the nose of the magnetosphere, showing the differential energy flux, $J_{\mathrm{E}}\left(E_{\mathrm{i}}, t_{\mathrm{s}}-t_{\mathrm{o}}\right)$ in spectrogram format, grey-scaled as a function of ion energy $E_{\mathrm{i}}$ and time elapsed since reconnection $\left(t_{\mathrm{s}}-t_{\mathrm{o}}\right)$ for sheath field clock angles $\theta_{\mathrm{sh}}$ of (a) $120^{\circ}$ and (b) $180^{\circ}$. (c) and (d). The corresponding simulated variations of the Doppler-shifted Lyman- $\alpha$ emission intensity $I$ that would be seen at the ionospheric footprint of that field line by the SI-12 imager channel of the FUV instrument on IMAGE. The interval $\Delta t$ is when the intensity $I$ exceeds a threshold $\left(I_{\text {peak }}-\Delta I\right)$, where $I_{\text {peak }}$ is the peak $I$ for the field line's light curve for the $\theta_{\mathrm{sh}}$ in question.

instrument response (Output O.5). Figures $4 \mathrm{a}$ and $4 \mathrm{~b}$ show the modeled precipitating cusp ion differential energy flux $J_{\mathrm{E}}$ on each newly opened field line reconnected at the nose of the magnetosphere, as a function of time elapsed since reconnection and ion energy, $E_{\mathrm{i}}$, for two specific clock angles between the reconnecting magnetosheath and magnetospheric field lines, $\theta_{\mathrm{sh}}=120^{\circ}$ and $\theta_{\mathrm{sh}}=180^{\circ}$. All other inputs to the model are the same in the two cases and are as given by LEA03. Comparison of these two cases reveals the effect of $\theta_{\mathrm{sh}}$, via its effect on the acceleration at the magnetopause and hence ion energy $E_{\mathrm{i}}$. Time elapsed since reconnection is $\left(t_{\mathrm{s}}-t_{\mathrm{o}}\right)$, where $t_{\mathrm{s}}$ is the time of observation (or, in this case, simulation) and $t_{\mathrm{o}}$ is the time that the field line observed/simulated was reconnected. Figures $4 \mathrm{c}$ and $4 \mathrm{~d}$ show the intensity of the consequent Doppler-shifted Lyman- $\alpha$ emission, convolved with the response of the FUV/SI-12 instrument on IMAGE, also as a function of $\left(t_{\mathrm{s}}-t_{\mathrm{o}}\right)$. Details of the procedure used to derive the differential energy flux spectra, $J_{\mathrm{E}}\left(E_{\mathrm{i}}, t_{\mathrm{s}}-t_{\mathrm{o}}\right)$, and the corresponding intensity variation, $I\left(t_{\mathrm{s}}-t_{\mathrm{o}}\right)$, are given above and in LEA03. Figure 4 shows that the peak intensity $I_{\text {peak }}$ is reached at $\left(t_{\mathrm{s}}-t_{\mathrm{o}}\right)$ near $300 \mathrm{~s}$ in both cases. Before this peak, the intensity increases as the precipitating ion flux increases. This is the effect of ion time-of-flight from the magnetopause to the ionosphere. After the peak, the intensity decays as both the flux and energy of the ions decrease due to a combination of the time-of-flight effects, magneto- sheath spatial structure and the decline in magnetopause ion acceleration (caused by the field line straightening as it evolves into the tail lobe). The cusp ion fluxes at the highest energies depend on the clock angle because of its effect on the ion acceleration on crossing the magnetopause and it is these fluxes which determine the proton emission intensity.

[12] In general, the draping of the IMF behind the bow shock means that the orientation of this magnetosheath field, for a given IMF clock angle $\theta$, is a complicated function of position on the magnetopause [Kobel and Flückiger, 1994]. However, the fact that the clock angle is preserved across the nose of the bow shock means that the clock angle over much of the dayside, $\theta_{\mathrm{sh}}$, is the same as that seen in interplanetary space, $\theta$ [Opgenoorth et al., 2001]. Hence for reconnection that is not too far removed from the nose, the variation with sheath clock angle of the modeled peak Lyman- $\alpha$ intensities, demonstrated by comparison of Figures $4 \mathrm{c}$ and $4 \mathrm{~d}$, can be compared to the observed variation of peak intensity (normalized to allow for the variations in the upstream solar wind density) as a function of IMF clock angle.

[13] Figure 5a shows modeled variations of intensity $I$ with $\left(t_{\mathrm{s}}-t_{\mathrm{o}}\right)$ for various constant $\theta_{\mathrm{sh}}$. For each clock angle, the times of peak intensity $\left(I=I_{\text {peak }}\right)$ and when $I=\left(\mathrm{e}^{-1} I_{\text {peak }}\right)$ are evaluated and hence the growth and decay time constants between $I_{\text {peak }}$ and the $\mathrm{e}^{-1} I_{\text {peak }}$ level are 



Figure 5. (a). Modeled Doppler-shifted Lyman- $\alpha$ intensity $I$, convolved with the response of the SI-12 channel of the IMAGE FUV instrument, as a function of time elapsed since reconnection $\left(t_{\mathrm{s}}-t_{\mathrm{o}}\right)$, for sheath field clock angles $\theta_{\mathrm{sh}}$ between $60^{\circ}$ and $180^{\circ}$ in steps of $10^{\circ}$. (b). The rise and decay time constants of the curves in Figure 5a, defined by the $\mathrm{e}^{-1} I_{\text {peak }}$ level, where $I_{\text {peak }}$ is the peak of each curve (see Figure 3d). The solid line is the sum of the rise and the decay times.

evaluated: these are shown by the dot-dash and dashed lines in Figure $5 \mathrm{~b}$. (Note that in this case, $\Delta I$ is equal to $I_{\text {peak }}\left(1-\mathrm{e}^{-1}\right)$ in Figure $\left.4 \mathrm{~d}\right)$. The duration for which the intensity exceeds the $\left(\mathrm{e}^{-1} I_{\text {peak }}\right)$ level, $\Delta t$, is the sum of these two time constants and is shown in Figure $5 \mathrm{~b}$ by the solid line. It can be seen that $\Delta t$ peaks at 4.8 min near $\theta_{\mathrm{sh}}=100^{\circ}$ and falls to just over 4 min near $\theta_{\mathrm{sh}}=180^{\circ}$.

[14] We here consider field lines reconnected at times $t_{\mathrm{o}}$ that are $15 \mathrm{~s}$ apart, the resolution of the IMF observations employed from the ACE satellite. Using the lagged ACE data, we know the solar wind proton concentration $N_{\mathrm{H}^{+}}$and clock angle $\theta$ of the field line reconnected (outside the bow shock) at that time $t_{\mathrm{o}}$. The propagation delays required are from the solar wind monitor to the reconnection site and so are smaller than those used by LEA03 by the $\approx 300 \mathrm{~s}$ between field line reconnection and $I$ attaining its peak value, $I_{\text {peak }}$ (see Figure 7 and associated discussion later in this section). At a later time $t_{\mathrm{s}}$, the elapsed time since reconnection is $\left(t_{\mathrm{s}}-t_{\mathrm{o}}\right)$ for that field line and the intensity is computed as in Figures $4 \mathrm{~b}, 4 \mathrm{~d}$, and $5 \mathrm{a}$. This gives the family of light curves shown in Figure 6a for the interval containing the observed brief swings to southward IMF. Since the model results are to be compared with the intensity of the brightest pixel recorded by the SI-12 instrument, $I_{\mathrm{o}}$, an envelope has been drawn around these curves to give $I_{\max }$, the brightest emission present at every $15 \mathrm{~s}$ time step in $t_{\mathrm{s}}$.

[15] The lower panel of Figure 6 shows $\left[t_{\mathrm{s}}-t_{\mathrm{o}}\right]_{\rho}$, the time since reconnection of the field line for which $I=I_{\max }$. If a given field line continues to be the one generating the peak intensity, $\left[t_{\mathrm{s}}-t_{\mathrm{o}}\right]_{\rho}$ ramps up as that open field line ages. The instantaneous drops in $\left[t_{\mathrm{s}}-t_{\mathrm{o}}\right]_{\rho}$ seen in Figure 6b occur when the intensity of what was the brightest field line is exceeded by the intensity of a more recently reconnected field line. Highlighted examples of such drops can be seen at times $t_{1}, t_{2}$, and $t_{3}$. The steady rise (with unity slope) seen between these times shows that a single field line governed the peak intensity, and thus the shape of the envelope, during the intervening periods. The step at time $t_{3}$ is considerably larger than the other highlighted steps. Before this, during a period of northward IMF, the intensity generated on the opened field lines failed to exceed the intensity on a field line opened previously while the IMF was southward. Only when the IMF turned southward again was sufficient intensity generated to exceed it and a large step in $\left[t_{\mathrm{s}}-t_{\mathrm{o}}\right]_{\rho}$ resulted. This panel serves to illustrate that the $I_{\max }$ envelope in Figure 6a represents the most intense field line, irrespective of where and when it was reconnected and that the variation of the peak observed intensity reflects the effects of the proton emission time constants shown in Figure $5 b$.

[16] An initial comparison of the modeled and observed intensities shows a clear dependence of emission intensity on the IMF clock angle, as found in the previous study by LEA03. As discussed above, the modeled peak intensity sequence $I_{\max }$ is at time resolution $15 \mathrm{~s}$. To make a quantitative comparison, we have sampled the $I_{\max }$ at the same rate as the FUV observations (every $122 \mathrm{~s}$ ) and found the cross correlation coefficient of the resulting sampled sequence, $I_{\mathrm{m}}$, with the observed peak intensity variation, $I_{\mathrm{o}}$. The lag was incremented in 15 -s steps for \pm 20 min about the nominal (predicted) value before $I_{\max }$ was sampled and so the data sequence $I_{\mathrm{m}}$ is not precisely the same for every lag. The results are shown in the correlogram in Figure 7a. In this figure, positive lag corresponds to $I_{\mathrm{o}}$ leading $I_{\mathrm{m}}$ and a lag of zero corresponds to the predicted ACE-to-ionosphere propagation delay employed by LEA03. The peak correlation is very high $(0.91)$, showing that the modeling is reproducing the observations closely. Using the autocorrelation functions of $I_{\mathrm{o}}$ and $I_{\mathrm{m}}$ to allow for the persistence in the data series [c.f. Lockwood, 2002], we find that this correlation is significant at the $98 \%$ level. The peak correlation is at a lag of $312 \mathrm{~s}$ which is almost all attributable to the fact that peak intensity is reached roughly $300 \mathrm{~s}$ after the field line is opened (see Figure 4): the nominal lag of LEA03 was between the IMF variations seen at ACE and the peak cusp aurora seen in the ionosphere, $\delta t_{\mathrm{E}}$. This is roughly $300 \mathrm{~s}$ greater than the ACE-to-magnetopause lag $\delta$ t required to generate the $I_{\max }$ and $I_{\mathrm{m}}$ modeled sequences. Thus the peak correlation at $312 \mathrm{~s}(5.2 \mathrm{~min})$ in Figure 7 confirms the lag variation determined by LEA03 is accurate. Figure $7 \mathrm{~b}$ analyses the uncertainty in 


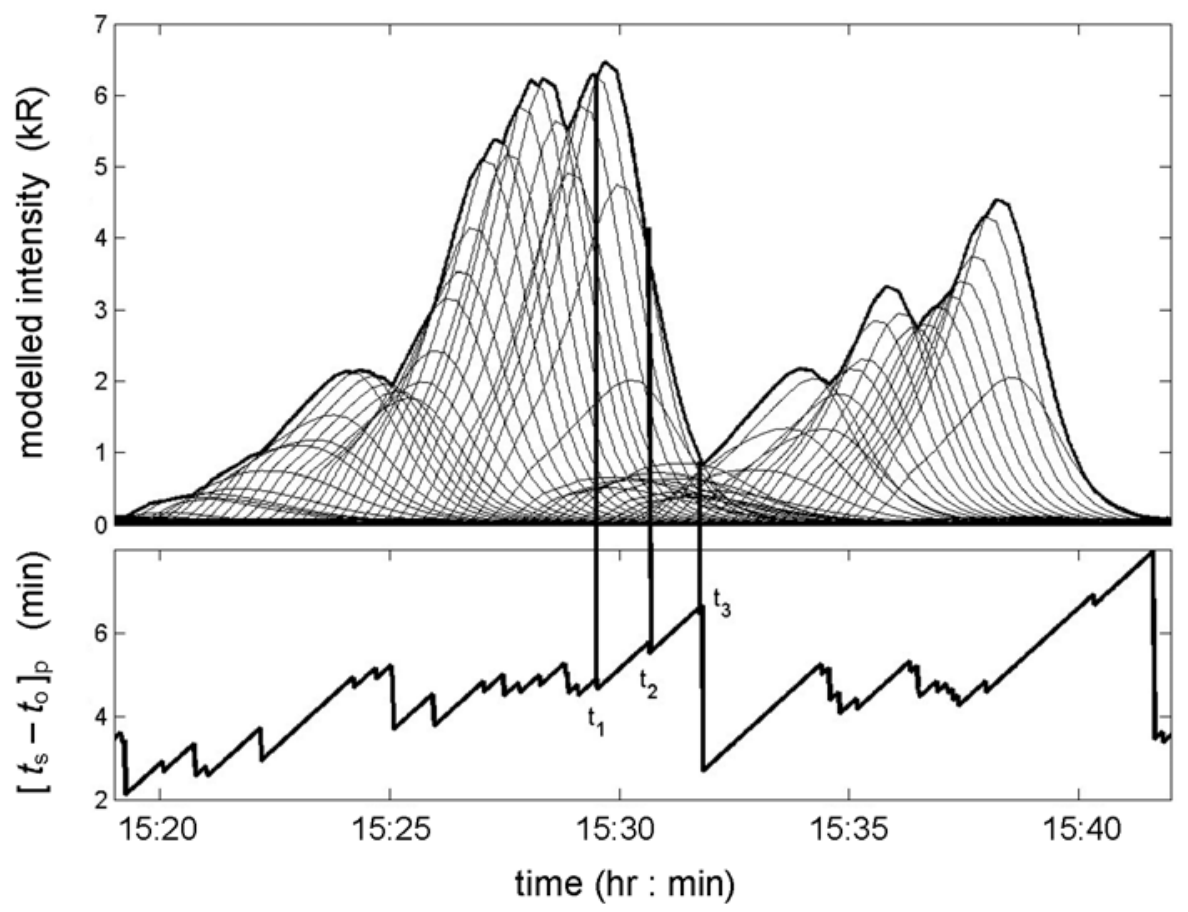

Figure 6. (a). Temporal variations of the Doppler-shifted Lyman- $\alpha$ intensity modeled on newly opened field lines that are reconnected at times $\mathrm{t}_{\mathrm{o}}$ that are $15 \mathrm{~s}$ apart, for the solar wind concentration $N_{\mathrm{sw}}$ and IMF clock angle $\theta$ observed by ACE at $\left(t_{\mathrm{o}}-\delta t\right)$, where $\delta t$ is the predicted ACE-to-magnetopause propagation delay. The thick line is $I_{\max }$, the envelope of the various light curves for these newly opened flux tubes. (b). The time elapsed since reconnection $\left[t_{\mathrm{s}}-t_{\mathrm{o}}\right]_{\rho}$ of the field line giving the peak intensity: times $t_{1}, t_{2}$, and $t_{3}$ are examples of downward steps in $\left[t_{\mathrm{s}}-t_{\mathrm{o}}\right]_{\rho}$ caused by a more recently reconnected flux tube surpassing an older flux tube in intensity.

the lag by looking at the significance $S$ of the difference between the correlation at a given lag and the peak correlation. $S$ is evaluated using the Fisher-Z test of the difference between two correlations [see Lockwood, 2002]. By definition, $S$ is zero at the peak correlation but increases as the correlation falls away from the peak. Figure $7 \mathrm{~b}$ shows that $S$ exceeds $90 \%$ at lags less than $3.6 \mathrm{~min}$ and exceeding $8.0 \mathrm{~min}$. Thus the uncertainty in the lag (at the $90 \%$ confidence level) is $\pm 2.2 \mathrm{~min}$. The linear regression fit between the optimally lagged modeled intensity $I_{\mathrm{m}}$ and the observed intensity $I_{\mathrm{o}}$ is shown in Figure 8 and can be used to scale the best fit model variation to the observations, the results being shown in Figure 9.

[17] It is noticeable in Figure 8 that the best regression fit is not a line with zero intercept and of unity slope: although the larger $I_{\mathrm{o}}$ and $I_{\mathrm{m}}$ values are comparable, $I_{\mathrm{o}}$ consistently exceeds $I_{\mathrm{m}}$ at low values. LEA03 explained this as the effect of lobe reconnection and the consequent northward-IMF cusp spot. Lobe reconnection generates a cusp spot because already-opened field lines are reconfigured so they thread the magnetopause closer to Earth than they did prior to reconfiguration, where sheath densities are higher. In addition, the tension force on these field lines accelerates them further sunward and accelerates injected ions towards the ionospheric cusp. However, one important additional reason why the northward IMF cusp spot is brighter than one might expect is because ionospheric convection is slower than for the southward-IMF case. This results in the ions being less dispersed and, when integrated over the energy range which excites the Doppler-shifted Lyman- $\alpha$ seen by FUV/SI-12, the flux is greater. Thus the northward-IMF spot tends to be smaller in size but relatively bright (considering that the sheath source plasma is further from the nose of the magnetopause and hence less dense). Figure 9 reveals that the model predicts a comparable peak intensity before and after the southward turnings of the IMF but that the observed peak Lyman- $\alpha$ intensities are larger/lower than the model predictions before/after the event. These disparities might be explained by a corresponding change in polarity of the IMF $B_{\mathrm{x}}$ component of the IMF during the event. Figure 10 shows the IMF components observed by ACE during the event. Before the interval of southward IMF the orientation was such that $B_{\mathrm{z}}>0$ and $B_{\mathrm{x}}<0$, whereas subsequently ACE observed $B_{\mathrm{z}}>0$ and $B_{\mathrm{x}}>0$. Lockwood and Moen [1999] have reasoned that the IMF $B_{\mathrm{x}}$ component can modify the tendency for lobe reconnection to take place in the summer hemisphere [Crooker and Rich, 1993]. Lockwood and Moen [1999] postulate that lobe reconnection occurs preferentially antisunward of the northern hemispheric cusp for $B_{\mathrm{z}}>0$ and $B_{\mathrm{x}}<0$, even during northern hemisphere winter. Thus the change in 


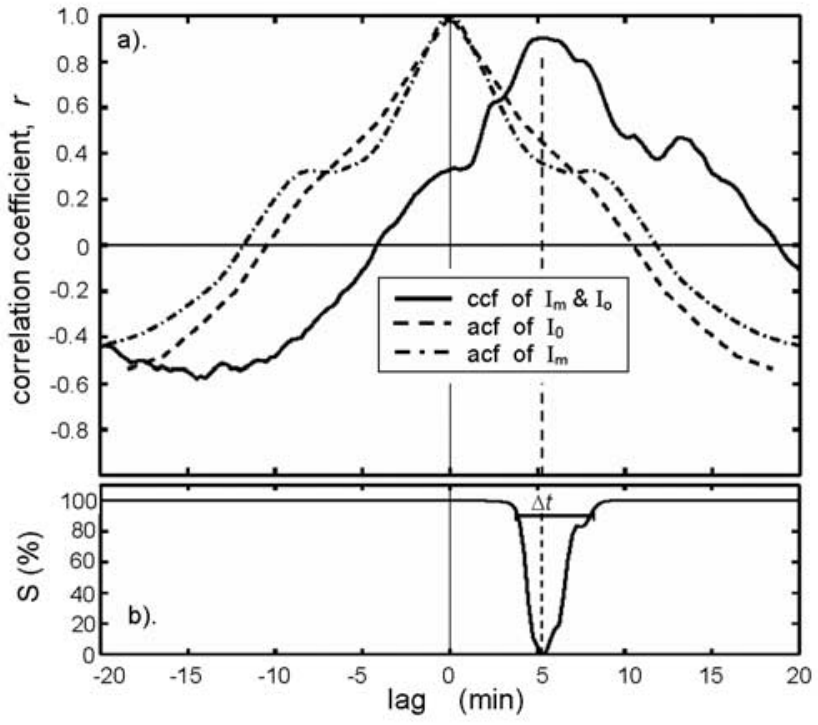

Figure 7. (a) Correlation analysis of the observed $\left(I_{\mathrm{o}}\right)$ and modeled-and-sampled $\left(I_{\mathrm{m}}\right)$ variations of peak intensity. $I_{\mathrm{m}}$ is the variation $I_{\max }$ shown in Figure 6a, sampled every $122 \mathrm{~s}$, the same interval as for the observed peak intensity, $I_{\mathrm{o}}$. The solid line shows the cross-correlation coefficient as a function of lag (the cross-correlation function, ccf), the dashed line shows the autocorrelation function (acf) of $I_{\mathrm{o}}$, and the dot-dash line shows the acf of $I_{\mathrm{m}}$. (b). The significance $S$ of the difference between a correlation at a given lag and the peak correlation.

IMF from $B_{\mathrm{x}}$ component from negative to positive moves the most likely lobe reconnection site from the northern hemisphere lobe before the event to the southern after it. Given that the IMAGE observations are in the northern hemisphere, this means the IMAGE is likely to have seen the effect of lobe reconnection before the event but not

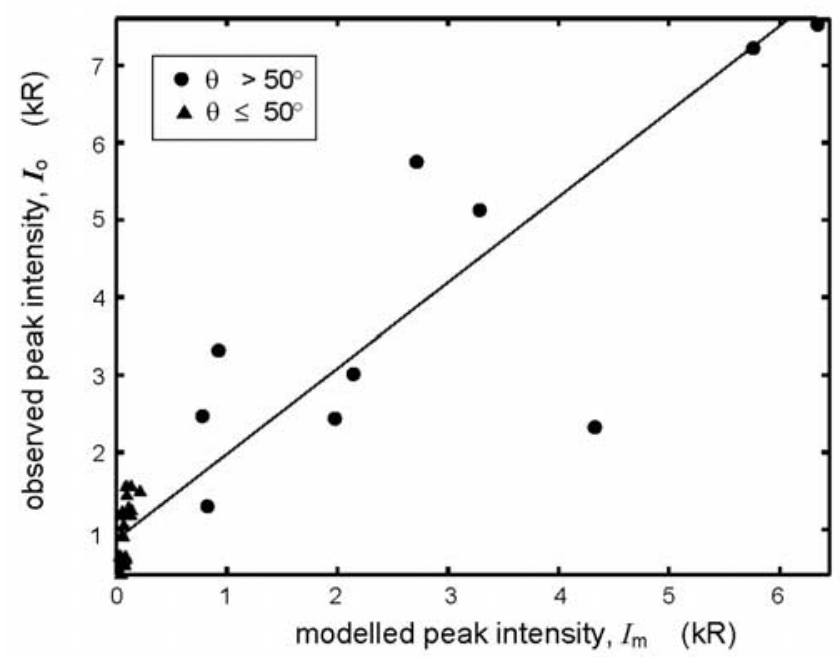

Figure 8. Scatter plot of $I_{\mathrm{o}}$ against $I_{\mathrm{m}}$ for the lag giving peak correlation shown in Figure 7 and the best-fit linear regression fit. Symbols differentiate between data taken when the IMF clock angle is smaller than or exceeds $50^{\circ}$.

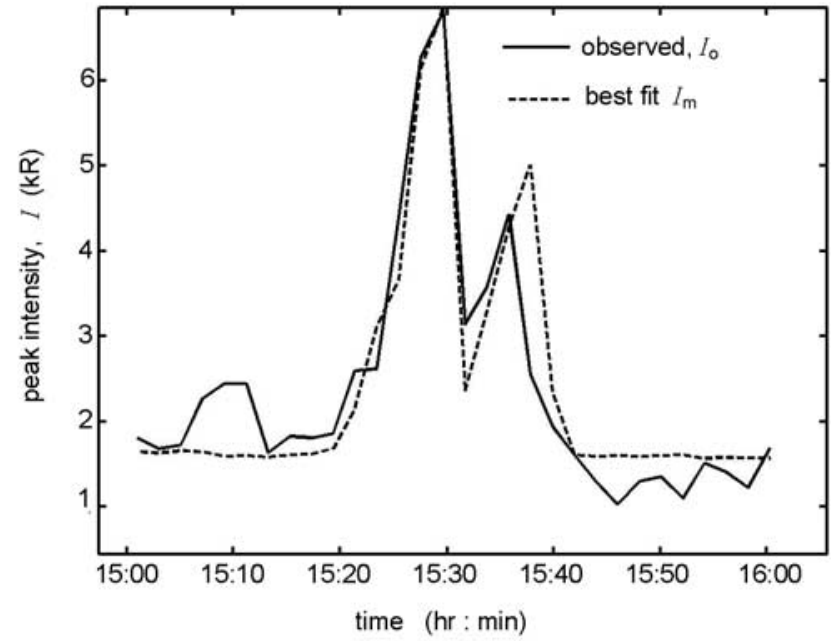

Figure 9. Comparison of peak Lyman- $\alpha$ intensity observed by the IMAGE FUV instrument, $I_{\mathrm{o}}$ (solid line) with the best-fit modeled variation, $I_{\mathrm{m}}$ (dashed line). $I_{\mathrm{m}}$ is the variation $I_{\max }$ shown in Figure 5, sampled every $122 \mathrm{~s}$ like the observations, at times which give the peak correlation shown in Figures 7 and 8.

after it. Thus the higher intensity seen before the interval of southward IMF (see Figure 9) is consistent with it being caused by magnetosheath particle entry facilitated by lobe reconnection.

\section{Spatial Distribution of the Proton Aurora}

[18] The LM04 model of ionospheric convection is based on the theory of the excitation of time-dependent ionospheric convection of Cowley and Lockwood [1992]; it predicts the pattern of ionospheric convection for general variations in reconnection rate, in both time and space, in both the low-latitude magnetopause and the cross-tail current sheet. Paper II describes how the inputs required by this model are defined for the interval studied here and analyzes their implications for the excitation of ionospheric convection. The variation of the tangential electric field along the dayside open-closed field line boundary (OCB) in the ionosphere, corresponding to the magnetopause reconnection rate, is defined using the observed, lagged IMF clock angle. The extent of the merging gap footprint of the magnetopause reconnection X-line is estimated from the extent of the proton aurora detected on the newly opened field lines by the FUV/SI-12 instrument, as shown in Figure 2. Other important inputs to the model are determined by matching model predictions and observed values of the invariant latitude of the noon open-closed field line boundary, $\Lambda_{\mathrm{OCB}}$, and of the transpolar voltage $\Phi_{\mathrm{PC}}$ (monitored by the IMAGE FUV imager and the SuperDARN HF radar network, respectively). Note that the LM04 model in its present form deals with the transfer of magnetic flux into and out of the open field line polar cap by low-latitude reconnection; it does not include effects taking place within the polar cap. Thus the east-west flows on newly-opened field lines, caused by the Svalgaard-Mansurov effect and associated with the Y-component of the IMF, are not 


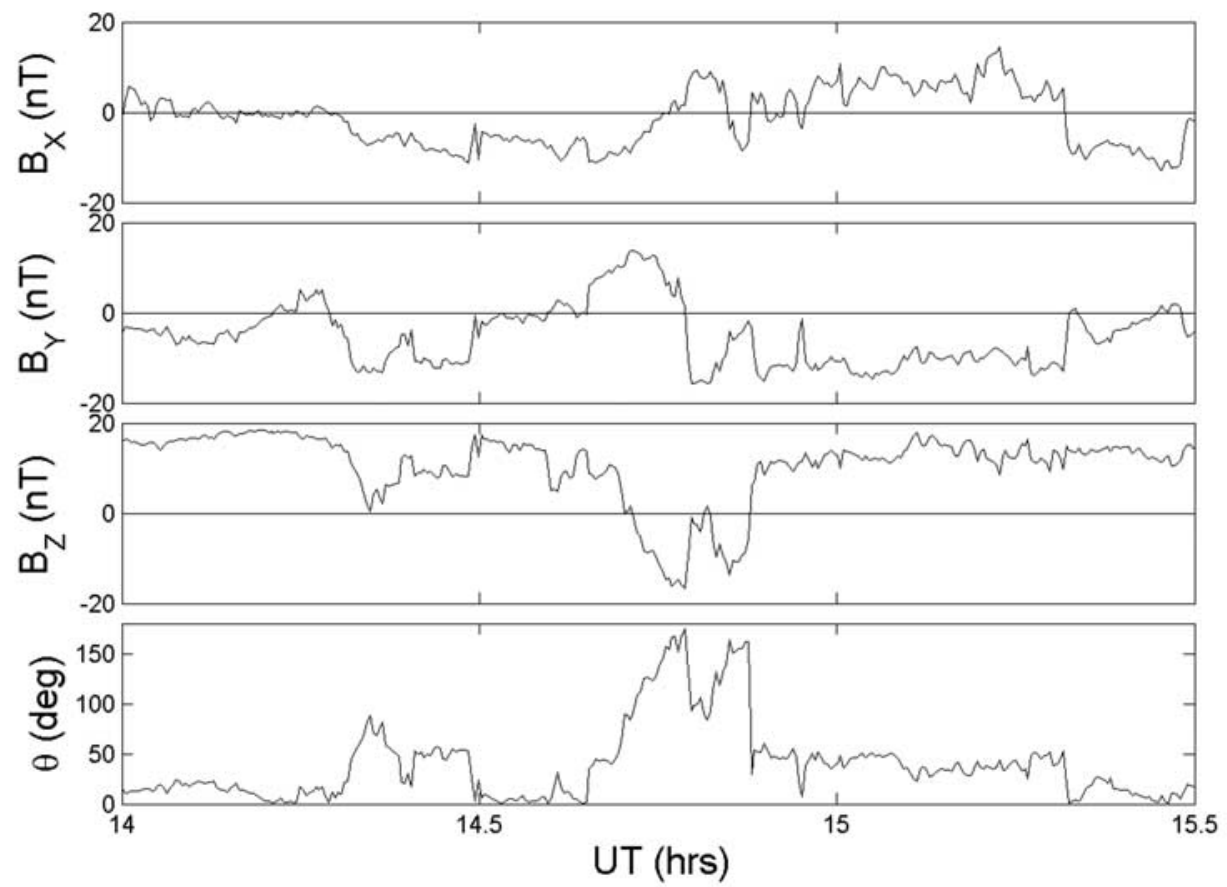

Figure 10. The interplanetary magnetic field components observed by ACE during the event in Geocentric Solar Magnetospheric (GSM) coordinates. From top to bottom: the $\mathrm{B}_{\mathrm{X}}, \mathrm{B}_{\mathrm{Y}}$, and $\mathrm{B}_{\mathrm{Z}}$ components and the clock angle $\theta$. All are shown as a function of the time of observation at ACE.

included nor is any stirring of polar cap caused by highlatitude (lobe) reconnection in one hemisphere. (However, any field line closure by lobe reconnection in both hemispheres could be included as a reverse-polarity tangential electric field along the dayside OCB). Quantitative analysis of the observed and modeled strength of flow is presented in Paper II.

[19] Paper II presents model flow patterns for this event, which reveal the evolution of patches of newly opened flux (see Figure 9 of Paper II). These patches are associated with equatorward erosion of the dayside OCB, caused by enhanced magnetopause reconnection and are highlighted in more detail in Figure 11 of the present paper, which shows the predicted intensity of Doppler-shifted Lyman- $\alpha$ on these newly opened field lines. The LM04 convection model allows us to track the motion of all the newly opened field lines, opened at time $t_{\mathrm{o}}$, so that their locations at every simulation time, $t_{\mathrm{s}}$, are known. Thus the model generates maps of time elapsed since reconnection $\left\{t_{\mathrm{s}}-t_{\mathrm{o}}\right\}$ in the MLT- $\Lambda$ frame. From the solar wind clock angle $\theta$ and plasma concentration $N_{\text {sw }}$ on the field line reconnected at time $t_{\mathrm{o}}$, the proton aurora intensity can be estimated for all $t_{\mathrm{S}}>t_{\mathrm{o}}$, as in Figure 6 . Thus we generate a map of the proton aurora intensity, as would be detected by FUV/SI-12 at any one time $t_{\mathrm{s}}$.

[20] The results are shown in Figure 11 for the times of the images shown in Figure 2. The field-of-view of each panel has been reduced to show the region of newly opened flux in greater detail. This region can be put into the global context using Figure 9 of Paper II. The panels correspond to the times of the images in Figure 2. It can be seen that the model is reproducing the general behavior of the FUV/SI-12 observations, with the first intensification reproduced exceptionally well. The largest discrepancy is at 1536, and this corresponds to the worst mismatch in the time series shown in Figure 9, where the observed second peak in $I_{\mathrm{o}}$ is slightly earlier and weaker than that in the model prediction, $I_{\mathrm{m}}$. This point is also the one major outlier in the scatter plot in shown in Figure 8. This difference may, at least in part, be caused by the polarity change in the IMF $B_{\mathrm{Y}}$ component and the observed shift of the proton aurora peak towards the morning sector. As discussed above, such effects are not included in the model.

\section{Time Constants of Proton Emission}

[21] The autocorrelation function (acf) of the observed intensity $I_{\mathrm{o}}$ is shown by the dashed line in Figure $7 \mathrm{a}$ and reveals a correlation time of order $10 \mathrm{~min}$. However, this may be set by the persistence of the emission, but could also reflect the persistence of the controlling IMF conditions. Figure 1 shows that $I_{\mathrm{o}}$ decays with a time constant of about 5 min following the abrupt decrease in IMF clock angle (seen at about 1540 in Figure 1). This is a better estimate of the persistence of the emissions and is close to the decay times predicted in Figure 5b. This, and the good match between the modeled and observed peak intensities shown in Figure 9 along with the general reproduction of the spatial pattern of proton aurora in Figure 11, gives great credence to the modeled variations of Doppler-shifted Lyman- $\alpha$ intensity, as shown in Figure 5a, and the rise and decay times shown in Figure 5b. As discussed in the introduction, these time constants smooth the effect of reconnection rate variations in the proton emissions. In this 
$15: 15: 18$

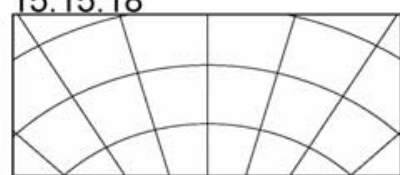

$15: 25: 32$

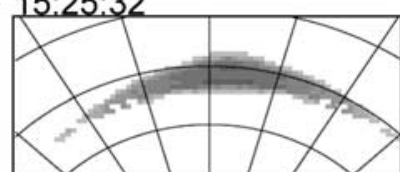

$15: 33: 43$

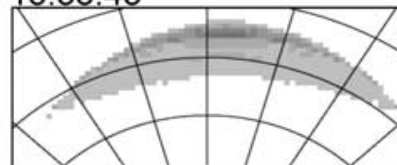

$15: 41: 53$

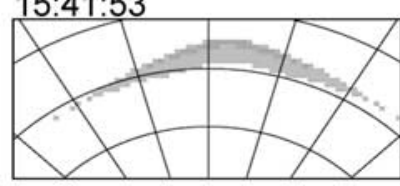

$15: 19: 24$

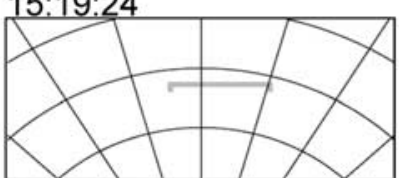

$15: 27: 35$

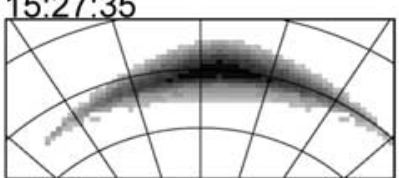

$15: 35: 45$

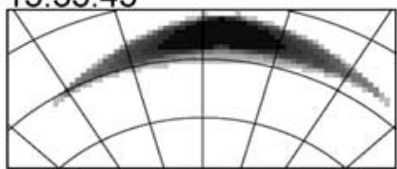

15:43:56

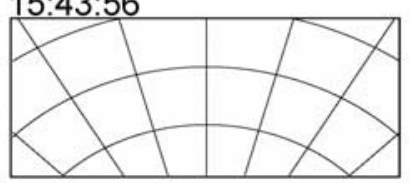

$15: 21: 26$

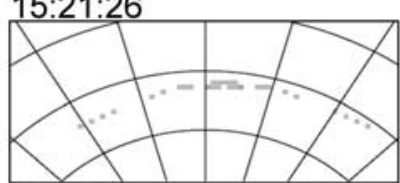

$15: 29: 37$

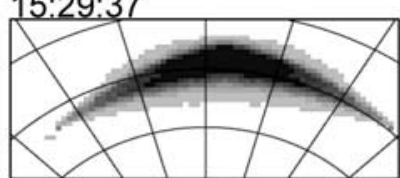

$15: 37: 48$

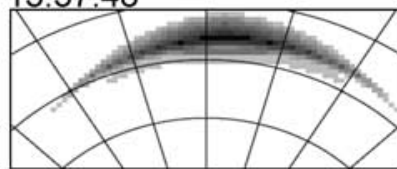

15:45:59

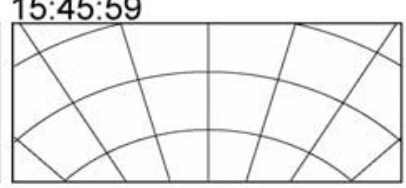

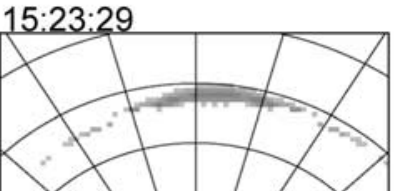

15:31:40

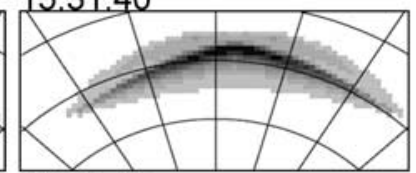

15:39:51
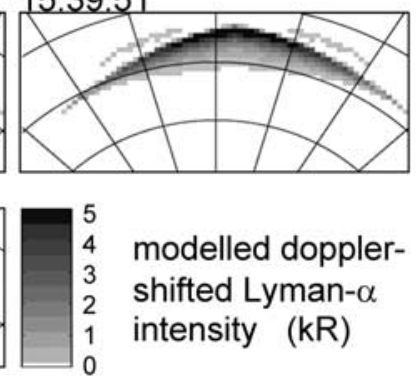

modelled doppler-

shifted Lyman- $\alpha$

intensity $(\mathrm{kR})$

Figure 11. Simulated maps of the Doppler-shifted Lyman- $\alpha$ emission intensity $I$ that would be detected by the SI-12 channel of the IMAGE FUV instrument. Panels are for 1516-1546 and are for the same times as the SI-12 images presented in Figure 2. The radial lines are for constant MLT, 1 hour apart with 12 MLT up the page; invariant latitudes of $75^{\circ}, 70^{\circ}$, and $65^{\circ}$ are shown.

section, we investigate the limits that this places on the detection of reconnection pulses. The observations presented in the previous section show two clear brightenings and expansions of the cusp proton aurora in response to two reconnection pulses. However, these pulses were directly driven by southward swings of the IMF and, for component reconnection, the effect of the reconnection rate pulse is convolved with the clock angle effect on the ion acceleration at the magnetopause. LEA03 discuss how antiparallel reconnection would require motions of the reconnection site (to locations where the magnetosheath ion concentration and temperature are different) to generate the same effect. In this section, we use the model to investigate the effect of reconnection pulses at various constant IMF clock angles.

[22] In general, the reconnection rate will show variations over a background level. Decreases of the rate to a low background value will cause steps in the cusp ion dispersion [e.g., Lockwood and Davis, 1996] and several observed examples of cusp ion steps have been interpreted in terms of such temporal variations because they are associated with poleward moving events and because the step propagates with the convection flow [Lockwood, 1995a, 1995b]. If the reconnection rate falls to a low (but nonzero) value, the step is not instantaneous, although it may appear to be if the ion spectra are not sampled sufficiently rapidly. If the reconnection does fall to zero between the pulses, the step is instantaneous, whatever the resolution of the ion data.

[23] If the reconnection rate does not fall to zero between any pulses, newly opened field lines are always being generated. The intensity of the proton aurora produced at the ionospheric footprint of each of these field lines will then evolve as shown in Figure 5a, and for constant IMF clock angle $\theta$ and solar wind concentration $N_{\text {sw }}$ the peak intensity $I_{\max }$ (and hence the sampled peak intensity $I_{\mathrm{m}}$ ) will be constant. However, because the amount of newly opened flux present will vary, the area of the proton aurora patch will vary. Thus detection of reconnection rate pulses over a steady background will require resolution of changes in the area of the proton aurora patch but will not involve any "blinking" of the patch on and off.

[24] On the other hand, if the reconnection rate does go to zero between pulses, the peak proton aurora intensity $I_{\max }$ is not constant and will start to show minima. We here investigate the depth and duration of these minima in order to evaluate if, and when, they could and should be detected. Figure $4 \mathrm{~d}$ defines the time $\Delta t$ for which the intensity on a given field line exceeds a threshold that is the peak intensity, minus a difference $\Delta I$. If we can detect a decrease in intensity greater than or equal to $\Delta I$, we will see the effect of an interval of zero reconnection rate, provided that it exceeds $\left(\Delta t+t_{\mathrm{R}}\right)$ in duration. The additional time $t_{\mathrm{R}}$ is the sampling interval of the data and must be added to $\Delta t$ to ensure that at least one data point is obtained when the intensity is below the threshold. We here use $t_{\mathrm{R}}=122 \mathrm{~s}$, appropriate to the FUV instrument.

[25] Figure 12a shows the predicted variation of $\left(\Delta t+t_{\mathrm{R}}\right)$ with sheath field clock angle $\theta_{\text {sh }}$ for a very high solar wind concentration $\left(N_{\mathrm{sw}}=30 \mathrm{~cm}^{-3}\right)$ for $\Delta I$ between 0.5 and $2.5 \mathrm{kR}$ (in steps of $0.25 \mathrm{kR}$ ). It can be seen that $\left(\Delta t+t_{\mathrm{R}}\right)$ values decrease as $\Delta I$ decreases and as $\theta_{\text {sh }}$ increases. Thus, for example, if we can detect intensity changes $\Delta I$ of $2.5 \mathrm{kR}$ and greater, then for this $N_{\mathrm{sw}}$ we can detect periods of zero reconnection of duration $5.5 \mathrm{~min}$ at $\theta_{\mathrm{sh}}$ of $180^{\circ}$. If the intensity resolution available is $\Delta I$ of $0.5 \mathrm{kR}$, this value is reduced to $3.5 \mathrm{~min}$. Note for component reconnection, with a lower $\theta_{\mathrm{sh}}$, these durations are increased. Figure $12 \mathrm{~b}$ shows 

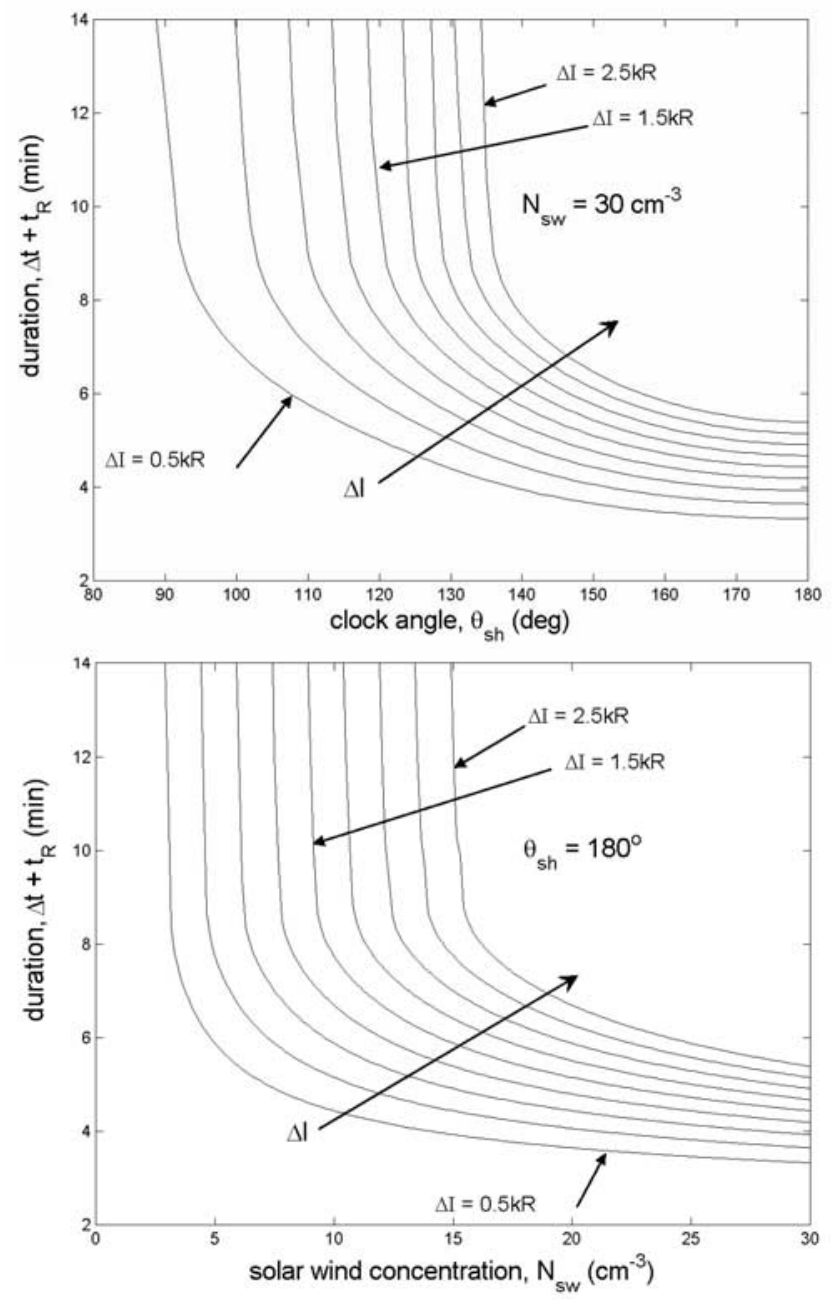

Figure 12. Modeled variations of $\left(\Delta t+t_{\mathrm{R}}\right)$ for $\Delta I$ between $0.5 \mathrm{kR}$ and $2.5 \mathrm{kR}$, in steps of $0.25 \mathrm{kR}$, where $\Delta t$ and $\Delta I$ are defined in Figure $4 \mathrm{~d}$ and $t_{\mathrm{R}}=2 \mathrm{~min}$. (a). As a function of sheath field clock angle $\theta_{\text {sh }}$ for constant solar wind concentration $N_{\mathrm{sw}}$ of $30 \mathrm{~cm}^{-3}$. (b). As a function of $N_{\mathrm{sw}}$ for constant $\theta_{\mathrm{sh}}$ of $180^{\circ}$.

the dependence of $\left(\Delta t+t_{\mathrm{R}}\right)$ as a function of $N_{\mathrm{sw}}$ for a constant $\theta_{\mathrm{sh}}$ of $180^{\circ}$ (antiparallel reconnection) for the same $\Delta I$ values. It can be seen that $\left(\Delta t+t_{\mathrm{R}}\right)$ increases as $N_{\mathrm{sw}}$ decreases. For the most common value of $N_{\mathrm{sw}}$ of $5 \mathrm{~cm}^{-3}$ [Hapgood et al., 1991], only $\Delta I$ of $0.5 \mathrm{kR}$ and $0.75 \mathrm{kR}$ will allow detection of the intensity changes and only for intervals of zero reconnection exceeding $5.5 \mathrm{~min}$ and $7.5 \mathrm{~min}$, respectively. Nevertheless, periods of zero reconnection between pulses should be detectable at high sheath field clock angles and high solar wind concentrations and Figures $12 \mathrm{a}$ and $12 \mathrm{~b}$ provide a means of evaluating when pulsed reconnection should be detected.

\section{Discussion and Conclusions}

[26] Combined with Paper II, the observations and modeling presented here provide compelling evidence for reconnection pulses which drive time-dependent responses with enhanced ionospheric flow and enhanced proton aurora. In this case, the pulses are directly driven by two isolated swings of the IMF toward a southward orientation that are roughly $10 \mathrm{~min}$ apart and thus do not represent spontaneous "internal" reconnection pulses for steady interplanetary conditions. The separation of $10 \mathrm{~min}$ allows us to distinguish the two separate responses in this case, but the persistence of the cusp precipitation and proton aurora (and of the flows; see Paper II) means that we will not always be able to isolate the effects of pulses, particularly if they are closer together in time.

[27] The excellent agreement shown in Figure 9 between $I_{\mathrm{o}}$, the peak Lyman- $\alpha$ intensity observed by the IMAGE FUV instrument, and $I_{\mathrm{m}}$, the best-fit modeled variation, highlights the clock angle dependence of the emission intensity seen in the cusp region. In LEA03, this dependence was discussed by showing the intensity of the cusp aurora as a function of the simultaneous IMF clock angle $\theta$ and solar wind density $N_{\mathrm{sw}}$. We have improved these findings by allowing for the evolution of the Dopplershifted Lyman- $\alpha$ emission with time since reconnection, the variation of that evolution with $\theta$ and $N_{\mathrm{sw}}$, and the limited sampling by the FUV instrument. The results presented here are generally similar to those of LEA03 but the scatter in the data is reduced and the correlation increased.

[28] The southward turning of the IMF reaching the ionosphere triggered the migration equatorward and intensification of the proton aurora near noon. As the polar cap expanded, the convection pattern intensified and the equipotentials migrated away from noon as the polar cap voltage, $\Phi_{\mathrm{PC}}$, increased. The dayside bulge in proton aurora is replicated by the model and is caused by the equatorward erosion of the OCB at the footprint of the magnetopause $\mathrm{X}$-line. Figure 11 predicts in detail how the bulge evolved, growing in longitude and expanding equatorward. The double nature of the intensification, caused by the IMF clock angle variation, is clear and is well reproduced by the model, although it predicts a slightly larger and later second peak than is actually observed.

[29] The success of the model in explaining the evolution of the cusp proton aurora, in peak intensity, location, and extent gives strong support to the rise and decay times of the emission predicted by the model and the persistence of proton emission of each newly opened field line. From this, we have studied the conditions required to observe pulsed magnetopause reconnection using the FUV/SI-12 instrument during southward IMF conditions.

[30] If the reconnection voltage is pulsed over a nonzero background level, for constant solar wind and IMF conditions, the peak proton emission intensity will not vary with time and the only signature will be lagged variations in the area of the patch of proton emission with the same periodicity as the reconnection voltage variation. Observations of cusp ion steps imply a wide range of interpulse durations [Lockwood et al., 1998]. If the reconnection rate falls to zero between the pulses, decreases in the peak emission will be observed in addition to the area changes, again with the periodicity of the reconnection variations. This "blinking" of the cusp proton aurora should be detectable in the case of antiparallel reconnection if the solar wind plasma concentration is high. If we take an 
intensity difference of $1 \mathrm{kR}$ to be resolvable above noise fluctuations, Figure 12b shows that for a very high solar wind concentrations of $N_{\mathrm{sw}}=30 \mathrm{~cm}^{-3}$, intervals of zero reconnection exceeding 4 min should be detected. For lower solar wind concentrations, intervals of zero reconnection will need to be longer if they are to be detected: for $N_{\mathrm{sw}}=$ $10 \mathrm{~cm}^{-3}$, intervals would need to be at least $6 \mathrm{~min}$ in duration and for the mode $N_{\mathrm{sw}}$ of $5 \mathrm{~cm}^{-3}$, no intensity variations would be detected at all. Figure 12a shows that for component reconnection, smaller clock angles between the reconnecting field lines would mean that only longer intervals of zero reconnection would be detected. Thus the FUV/SI-12 instrument allows us to place limits on intervals of zero reconnection between pulses. It should be stressed that this analysis is for southward IMF conditions. As pointed out by Frey et al. [2003], the same sort of considerations would apply to lobe reconnection during northward IMF, when the reversed ion dispersion often observed can have otherwise very similar characteristics to those during southward IMF [Woch and Lundin, 1992]; however, the relevant time constants and the limits placed would be different because of the differences in the location of the reconnection site and because the evolution of the recently reconnected field lines would be different.

[31] Acknowledgments. The authors are grateful to Norman F. Ness (Bartol Research Institute) and the MAG team for provision of the ACE IMF data and David J. McComas (LANL) and the SWEPAM team for provision of the ACE solar wind data. We also thank all participants in the SuperDARN radar network for their contributions to the mapped potential radar data and the IMAGE-SMOC team who keep the IMAGE spacecraft running. The IMAGE FUV investigation was supported by NASA through SwRI subcontract 83820 at the University of California, Berkeley, under contract NAS5-96020. The CUTLASS SuperDARN radar and the work of ML is funded by the UK Particle Physics and Astronomy Research Council (PPARC). The work of KT and SKM was supported by a Southampton University Ph.D. studentship and a PPARC studentship, respectively.

[32] Arthur Richmond thanks Delores J. Knipp and another reviewer for their assistance in evaluating this paper.

\section{References}

Burch, J. L. (1985), Quasi-neutrality in the polar cusp, Geophys. Res. Lett., $12,469-472$.

Chisham, G., M. Pinnock, I. J. Coleman, M. R. Hairston, and A. D. M. Walker (2002), An unusual geometry of the ionospheric signature of the cusp: Implications for magnetopause merging sites, Ann. Geophys., 20, $29-40$.

Coleman, I. J., M. Pinnock, and A. S. Rodger (2000), The ionospheric footprint of anti-parallel merging regions on the dayside magnetopause, Ann. Geophys., 18, 511-516.

Coleman, I. J., G. Chisham, M. Pinnock, and M. P. Freeman (2001), An ionospheric convection signature of anti-parallel reconnection, J. Geophys. Res., 106, 28,995-29,008.

Cowley, S. W. H. (1982), The causes of convection in the Earth's magnetosphere: A review of developments during IMS, Rev. Geophys., 20, $531-$ 565 .

Cowley, S. W. H., and M. Lockwood (1992), Excitation and decay of solarwind driven flows in the magnetosphere-ionosphere system, Ann Geophys., 10, 103-115.

Crooker, N. U. (1979), Dayside merging and cusp geometry, J. Geophys. Res., 84, 951-959.

Crooker, N. U., and F. J. Rich (1993), Lobe cell convection as a summer phenomenon, J. Geophys. Res., 98, 13,403-13,407.

Crooker, N. U., F. Toffoletto, and M. S. Gusenhoven (1991), Opening the cusp, J. Geophys. Res., 96, 3497-3503.

Davis, C. J., and M. Lockwood (1996), Predicted signatures of pulsed reconnection in ESR data, Ann. Geophys., 14, 1246-1256.

Frey, H. U., S. B. Mende, C. W. Carlson, G.-C. Gérard, B. Hubert, I. Spann, R. Gladstone, and T. J. Immel (2001a), The electron and proton aurora seen by IMAGE-FUV and FAST, Geophys. Res. Lett., 28 , $1135-1139$
Frey, H. U., S. B. Mende, T. J. Immel, S. A. Fuselier, E. S. Claflin, J.-C. Gérard, and B. Hubert (2001b), Proton aurora in the cusp, J. Geophys. Res., 107(A7), 1091, doi:10.1029/2001JA900161.

Frey, H. U., T. D. Phan, S. A. Fuselier, and S. B. Mende (2003), Continuous magnetic reconnection at Earth's magnetopause, Nature, 426, 533-537, doi:10.1038/nature02084

Gérard, J.-C., B. Hubert, M. Meurant, V. I. Shematovich, D. V. Bisoikalo, H. U. Frey, S. Mende, and G. R. Gladstone (2001), Observations of the proton aurora with IMAGE FUV imager and simultaneous ion flux measurements, J. Geophys. Res., 106, 28,939-28,948.

Gonzalez, W. D., and F. S. Mozer (1974), A quantitative model for the potential resulting from reconnection with an arbitrary interplanetary magnetic field, J. Geophys. Res., 79, 4186-4194.

Hapgood, M. A., G. Bowe, M. Lockwood, D. M. Willis, and Y. Tulunay (1991), Variability of the interplanetary magnetic field at 1 A.U. over 24 years: 1963-1986, Planet. Space Sci., 39, 411423.

Hill, T. W., and P. H. Reiff (1977), Evidence of magnetospheric cusp proton acceleration by magnetic merging at the dayside magnetopause, J. Geophys. Res., 82, 3623-3628.

Kobel, E., and E. O. Flückiger (1994), A model of the steady state magnetic field in the magnetosheath, J. Geophys. Res., 99, 23,61723,622 .

Lockwood, M. (1995a), Ground-Based and Satellite Observations of the Cusp: Evidence for Pulsed Magnetopause Reconnection, in Physics of the Magnetopause, Geophys. Monogr. Ser., vol. 90, edited by P. Song, B. U. O. Sonnerup, and M. F. Thomsen, pp. 417-426, AGU, Washington, D. C.

Lockwood, M. (1995b), The location and characteristics of the reconnection $\mathrm{X}$-line deduced from low-altitude satellite and ground-based observations: 1. Theory, J. Geophys. Res., 100, 21,791-21,802.

Lockwood, M. (1997), Energy and pitch angle dispersions of LLBL/cusp ions seen at middle altitudes: predictions by the open magnetosphere model, Ann. Geophys., 15, 1501-1514.

Lockwood, M. (2002), An evaluation of the correlation between open solar flux and total solar irradiance, Astron. Astrophys., 382, 6786361.

Lockwood, M., and C. J. Davis (1995), The occurrence probability, width and number of steps of cusp precipitation for fully-pulsed reconnection at the dayside magnetopause, J. Geophys. Res., 100, $7627-7640$.

Lockwood, M., and C. J. Davis (1996), On the longitudinal extent of magnetopause reconnection bursts, Ann. Geophys., 14, 865-878.

Lockwood, M., and M. A. Hapgood (1998), On the cause of a magnetospheric flux transfer event, J. Geophys. Res., 103, $26,453-26,478$

Lockwood, M., and J. Moen (1999), Reconfiguration and closure of lobe flux by reconnection during northward IMF: evidence for signatures in cusp/cleft auroral emissions, Ann. Geophys., 17, 9961011.

Lockwood, M., and S. K. Morley (2004), A numerical model of the ionospheric signatures of time-varying magnetic reconnection: I. Ionospheric convection, Ann. Geophys., 22, 73-91.

Lockwood, M., and M. F. Smith (1992), The variation of reconnection rate at the dayside magnetopause and cusp ion precipitation, J. Geophys. Res., $97,14,841-14,847$.

Lockwood, M., C. J. Davis, T. G. Onsager, and J. A. Scudder (1998), Modelling signatures of pulsed magnetopause reconnection in cusp ion dispersion signatures seen at middle altitudes, Geophys. Res. Lett., 25, 591-594.

Lockwood, M., B. S. Lanchester, H. U. Frey, K. Throp, S. K. Morley, S. E. Milan, and M. Lester (2003), IMF control of cusp proton emission intensity and dayside convection: Implications for component and anti-parallel reconnection, Ann. Geophys., 21, 955982.

Lockwood, M., B. S. Lanchester, S. K. Morley, K. Throp, S. E. Milan, and H. U. Frey (2005), Modeling the observed proton aurora and ionospheric convection responses to changes in the IMF clock angle: 2 . The persistence of ionospheric convection, J. Geophys. Res., doi:10.1029/ 2003JA010307, in press.

Mende, S. B., H. Heetderks, and H. U. Frey (2000a), Far ultraviolet imaging from the IMAGE spacecraft, 1. System design, Space Sci. Rev., 91, 243-270.

Mende, S. B., H. Heetderks, and H. U. Frey (2000b), Far ultraviolet imaging from the IMAGE spacecraft, 3. Spectral imaging of Lyman alpha and OI $135.6 \eta \mathrm{m}$, Space Sci. Rev., 91, 287-318.

Onsager, T. G., C. A. Kletzing, J. B. Austin, and H. MacKiernan (1993), Model of magnetosheath plasma in the magnetopshere: Cusp and mantle particles at low-altitudes, Geophys. Res. Lett., 20, 479482. 
Opgenoorth, H. J., et al. (2001), Coordinated ground-based, low altitude satellite and Cluster observations on global and local scales during a transient postnoon sector excursion of the magnetospheric cusp, Ann. Geophys., 19, 1367-1398.

Ruohoniemi, J. M., and K. B. Baker (1998), Large-scale imaging of highlatitude convection with Super Dual Auroral Radar Network HF radar observations, J. Geophys. Res., 103, 20,797-20,812.

Sibeck, D. G., R. E. Lopez, and E. C. Roelof (1991), Solar wind control of the magnetopause shape, location, and motion, J. Geophys. Res., 96, $5489-5495$.

Sonnerup, B. U. Ö. (1974), Magnetopause reconnection rate, J. Geophys. Res., 79, 1546-1549.

Spreiter, J. R., A. L. Summers, and A. Y. Alksne (1966), Hydromag netic flow around the magnetosphere, Planet. Space Sci., 14, $223-$ 253.

Tsyganenko, N. A. (1987), Global quantitative models of the geomagnetic field in the cislunar magnetosphere for different disturbance levels, Planet. Space Sci., 35, 1347-1358.
Woch, J., and R. Lundin (1992), Magnetosheath plasma precipitation in the polar cusp and its control by the interplanetary magnetic field, J. Geophys. Res., 97, 1421-1430.

H. U. Frey, Space Sciences Laboratory, University of California, Berkeley, Berkeley, CA 94720-7450, USA

B. S. Lanchester and K. Throp, Department of Physics and Astronomy, Southampton University, Highfield, Southampton, Hampshire, SO9 5NH, UK.

M. Lockwood, Space Science Department, Rutherford Appleton Laboratory, Chilton, Didcot, Oxfordshire, OX11 0QZ, UK. (m.lockwood@ rl.ac.uk)

S. K. Morley, CRC for Satellite Systems, School of Mathematics and Physical Sciences, University of Newcastle, Callaghan, New South Wales NSW 2308, Australia. 